\title{
Coordinación de competencias transversales en asignaturas de ámbito nuclear en el Grado de Ingeniero de la Energía
}

\section{Sergio Gallardo ${ }^{a}$, Sofía Carlos ${ }^{b}$, Sebastián Martorell ${ }^{c}$ y José Ródenas ${ }^{d}$}

aDepartamento de Ingeniería Química y Nuclear, Universitat Politècnica de València, Spain, email: sergalbe@iqn.upv.es; 'bepartamento de Ingeniería Química y Nuclear, Universitat Politècnica de València, Spain, email: scarlos@iqn.upv.es; 'Departamento de Ingeniería Química y Nuclear, Universitat Politècnica de València, Spain, email: smartore@iqn.upv.es; ${ }^{c}$ Departamento de Ingeniería Química y Nuclear, Universitat Politècnica de València, Spain, email: smartore@iqn.upv.es; ${ }^{\mathrm{d} D e p a r t a m e n t o ~ d e ~ I n g e n i e r i ́ a ~ Q u i ́ m i c a ~ y ~ N u c l e a r, ~ U n i v e r s i t a t ~ P o l i t e ̀ c n i c a ~ d e ~ V a l e ̀ n c i a, ~ S p a i n, ~ e m a i l: ~}$ jrodenas@iqn.upv.es;

\begin{abstract}
After the implantation of the new Bachelor and Master Degrees and in order to group different skills profiles, the Universitat Politècnica de València (UPV) defined a list of generic competences. This list consists of a total of 13 competences to be trained and assessed in different subjects. In this frame, coordination between subjects should be improved to ensure that all the compentences are considered in the Degree. The coordination must consider the design and the assessment of activities. In the nuclear field subjects, we propose to promote the relationship between subjects taking profit of the synergy between them. One way to achieve this goal is to design activities that are related to other subjects, so that the same transversal skill can be worked on one subject but establishing connections with others. Based on this premise, this paper presents the benefits of coordination in transverse skills and their impact on the integration of nuclear subjects.
\end{abstract}

Keywords: Transversal skill; Bachelor Degree in Energy Engineering; Nuclear field subjects.

\footnotetext{
Resumen

Después de la implantación de los nuevos Grados y Másteres, la Universitat Politècnica de València (UPV) publicó una lista de competencias genéricas para agrupar los diferentes perfiles competenciales. Esta lista consta de un total de 13 competencias que deben trabajarse y evaluarse en las diferentes materias. En este marco, se debe mejorar la coordinación entre las asignaturas para asegurar que todas las competencias se trabajan a lo largo de la titulación. La coordinación debe considerar el diseño y la evaluación de las actividades. En las asignaturas de ámbito nuclear, se propone potenciar la relación entre asignaturas aprovechando las sinergias que exiten entre ellas. Una manera de conseguirlo es diseñando actividades que estén relacionadas con otras asignaturas, de forma que una misma competencia transversal
} 
pueda trabajarse en una asignatura pero estableciendo conexiones con otras. Partiendo de esta premisa, en este trabajo se presentan los beneficios de la coordinación en materia de competencias transversales y su repercusión en la integración de las asignaturas de ámbito nuclear.

Palabras clave: competencia transversal, grado ingeniero de la energía, asignaturas de ámbito nuclear.

\section{Introducción}

Los actuales planes de estudio del Espacio Europeo de Educación Superior (EEES) incluyen una serie de competencias transversales y específicas que deben adquirir los alumnos al finalizar las distintas titulaciones (Sanz de Aceo, 2010). A través del proceso de acreditación Accreditation Board for Engineering and Technology (ABET) llevado a cabo en algunas titulaciones en la Universitat Politècnica de València (UPV), ha quedado patente la necesidad de evaluar y de obtener evidencias de las competencias, principalmente transversales, que deben trabajarse en las titulaciones en cuestión. En este contexto, la UPV definió las Competencias Transversales UPV (UPV, 2015a), con el fin de abordar el problema de trabajo y evaluación de las distintas competencias transversales en todas las titulaciones que imparte. El establecimiento de 13 competencias tranversales según la UPV, obliga a realizar un importante trabajo para asegurar que el alumno egresado haya trabajado de manera adecuada y balanceada todas ellas. La coordinación es esencial para poder asegurar este punto. Sin embargo, la coordinación no debe quedarse únicamente en el nivel de toda la titulación, bien sea grado o máster. La coordinación debe descender al nivel de las materias y al nivel de la optatividad. Un caso muy concreto es el relativo a las asignaturas de ámbito nuclear. La especificidad de contenidos obliga a redoblar los esfuerzos de coordinación para evitar solapes y asegurar un diseño, desarrollo y evaluación coherente de las competencias transversales. La coordinación exige realizar un ejercicio de síntesis de contenidos y mostrar de forma explícita la integración de las distintas asingaturas, en este caso del campo de conocimiento de nuclear.

\section{Objetivos}

Según lo expuesto en la introducción, parece necesario desarrollar instrumentos que faciliten la evaluación de las competencias y por tanto trasladar progresivamente el sistema tradicional a una "evaluación POR competencias", y además resulta imprescindible integrar dichas competencias en las asignaturas, plasmándolas en actividades.

El diseño, desarrollo y evaluación de actividades para trabajar estas competencias debe ser conocido por todos los profesores involucrados en el mismo campo de conocimiento, en este caso, nuclear.

(c) EY-NC-ND 2017, Universitat Politècnica de València 
En este contexto, el objetivo fundamental de este trabajo es estudiar la importancia de la coordinación en las asignaturas de ámbito nuclear en el Grado de Ingeniero de la Energía (GIE) para trabajar de manera efectiva e integrada diversas competencias transversales.

En un primer estadio, se ha establecido un grupo de trabajo que afecta únicamente a tres asignaturas de ámbito nuclear y de carácter optativo con el objetivo de poder exportar la experiencia al resto de asignaturas, incluidas las obligatorias (en $2^{\circ}$ y $4^{\circ}$ curso de GIE).

Los objetivos específicos de este trabajo son los siguientes:

1. Identificación, análisis y comprensión de competencias transversales comunes en algunas asignaturas de ámbito nuclear.

2. Diseño de actividades en las diferentes asignaturas, utilizando los mismos criterios en cuanto al significado de cada competencia.

3. Identificación de puntos comunes y sinergias entre asignaturas para poder trabajar diferentes competencias de distintas asignaturas en una misma actividad.

4. Establecimiento de indicadores para la evaluación mediante rúbricas, de forma que se potencie la interrelación con las otras asignaturas.

Este plan de trabajo redunda en la idea de mejora continua en el desarrollo y evaluación de las competencias transversales y pone el foco en las asignaturas de ámbito nuclear.

\section{Desarrollo de la innovación}

\subsection{Contexto}

Desde el punto de vista curricular, es esencial cubrir de manera completa y coherente todas las competencias tranversales de una titulación, bien sea a nivel de grado o de máster. En este contexto, la coordinación entre asignaturas es esencial no solo para evitar solapes y vacíos en los contenidos, si no además para reforzar la coherencia en el desarrollo y evaluación de las competencias transversales. La coordinación debe realizarse a nivel de titulación y materia, pero además debe realizarse a un nivel más profundo. En este trabajo se propone una coordinación de competencias transversales desde el punto de vista de un determinado campo: el campo de conocimiento nuclear. En el caso concreto de la ingeniería nuclear, el escenario que nos encontramos al analizar el Grado de Ingeniero de la Energía (GIE) es el mostrado en la Tabla 1.

Como se puede apreciar a partir de la Tabla 1, el contenido nuclear explícito se concentra en $4^{\text {o }}$ curso, especialmente en el bloque de optatividad del segundo cuatrimestre.

Tanto la asignatura "Energía y Desarrollo Sostenible" como la asignatura "Tecnología Nuclear" son obligatorias y por tanto, todos los alumnos matriculados en el grado deben cursarlas. Durante el tiempo en el que se ha impartido estas asignaturas, el número de matriculados ha permanecido relativamente constante, entre 75 y 85 alumnos. El caso de "Centrales Nucleares Avanzadas", "Seguridad Nuclear", "Operación de Reactores Nucleares" y "Protección Radiológica" es muy distinto. Se trata de asignaturas optativas y de un carácter marcadamente específico, por lo que el número de alumnos que eligen cursarlas en muy reducido. De hecho, el número medio de matriculados en los últimos cursos 
académicos ha sido de 3 o 4 alumnos en cada una por término medio. Sin embargo, y de manera totalmente justificada, los alumnos que deciden cursar alguna de estas asignaturas, suelen matricularse en alguna de las otras. En conclusión, debe existir cierta coherencia en cuanto al desarrollo, trabajo y evaluación de las competencias específicas y transversales en estas asignaturas.

Tabla 1. Asignaturas de ámbito nuclear en GIE

\begin{tabular}{ccccc}
\hline Asignatura & Curso & Cuatrimestre & Tipo & Créditos \\
\hline Energía y Desarrollo Sostenible & $2^{\circ}$ & B & Obligatoria & 6 \\
Tecnología Nuclear & $4^{\circ}$ & B & Obligatoria & 6 \\
Centrales Nucleares Avanzadas & $4^{\circ}$ & B & Optativa & 4,5 \\
Seguridad Nuclear & $4^{\circ}$ & B & Optativa & 4,5 \\
Operación de Reactores & $4^{\circ}$ & B & Optativa & 4,5 \\
Nucleares & & & & \\
Protección Radiológica & $4^{\circ}$ & B & Optativa & 4,5 \\
\hline
\end{tabular}

\subsection{Las competencias transversales en la optatividad nuclear}

En la Tabla 2 se muestra la asignación de puntos de control a las asignaturas de ámbito nuclear en GIE.

Como se puede apreciar, las competencias transversales de las cuales estas asignaturas son punto de control son básicamente "Comprensión e integración", "Aplicación y pensamiento práctico", "Responsabilidad ética, medioambiental y profesional", "Pensamiento crítico", "Conocimiento de problemas contemporáneos" e "Instrumental específica". Es importante señalar que el hecho de no ser punto de control, no implica que no se trabajen otras competencias transversales. Existe una gran concentración en cuanto a puntos de control en tres de las competencias transversales: "Comprensión e integración", "Conocimiento de problemas contemporáneos" e "Instrumental específica".

Si nos centramos en las asignaturas de carácter optativo, se observa que son punto de control preferentemente de "Comprensión e integración" e "Instrumental específica".

Es evidente que todas estas asignaturas, por su contenido y ámbito son especialmente aptas para ser punto de control en temas de compresión e integración de conocimientos y en temas de mejora de la capacidad en el uso de herramientas específicas.

Una vez caracterizado el contexto, parece claro que es importante aunar esfuerzos para mejorar la coherencia en el trabajo y evaluación de este reducido número de competencias transversales. 
Tabla 2. Competencias transversales en las ssignaturas de ámbito nuclear en GIE

\begin{tabular}{cl}
\hline Identificador & \multicolumn{1}{c}{ Asignatura } \\
\hline 1 & Energía y Desarrollo Sostenible \\
2 & Tecnología Nuclear \\
3 & Centrales Nucleares Avanzadas \\
4 & Seguridad Nuclear \\
5 & Operación de Reactores Nucleares \\
6 & Protección Radiológica \\
\hline
\end{tabular}

\begin{tabular}{|c|c|c|c|c|c|c|c|c|c|c|c|c|c|}
\hline 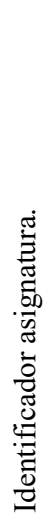 & 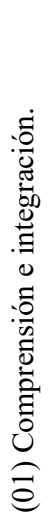 & 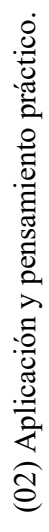 & 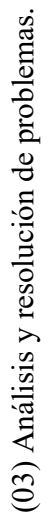 & 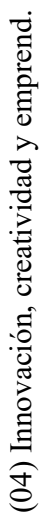 & 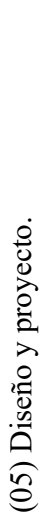 & 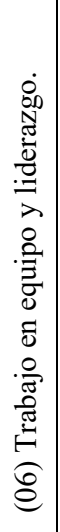 & 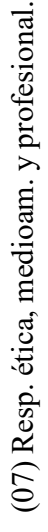 & 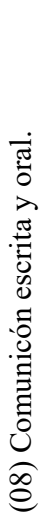 & 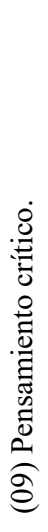 & 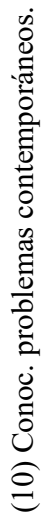 & 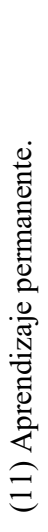 & 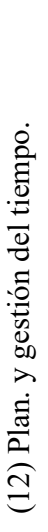 & 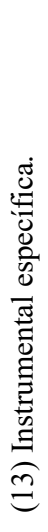 \\
\hline 1 & & & & & & & $\mathrm{x}$ & & & $\mathrm{x}$ & & & $\mathrm{x}$ \\
\hline 2 & & $\mathrm{x}$ & & & & & & & & $\mathrm{x}$ & & & $\mathrm{x}$ \\
\hline 3 & & $\mathrm{x}$ & & & & & & & $\mathrm{x}$ & $\mathrm{X}$ & & & \\
\hline 4 & $\mathrm{x}$ & & & & & & & & & & & & $\mathrm{X}$ \\
\hline 5 & $\mathrm{x}$ & & & & & & & & & & & & $\mathrm{x}$ \\
\hline 6 & X & & & & & & & & & & & & X \\
\hline
\end{tabular}

\subsection{Los niveles de dominio}

El siguiente problemas que se plantea se refiere a cómo se están trabajando actualmente muchas de esas asignaturas. En este trabajo nos vamos a centrar en las asignaturas de carácter optativo por una razón. Como se ha expuesto anteriormente, los alumnos de estas asignaturas optativas suelen elegir muchas de ellas, por lo que se puede disponer de una retroalimentación muy valiosa de cómo percibe la coherencia en la evaluación un mismo alumno en las diferentes asignaturas. En el proyecto institucional de competencias transversales que está llevando a cabo la Universitat Politècnica de València, se han identicado 13 competencias transversales y se han desarrollado rúbricas (UPV, 2015b) de evaluación genéricas para los 
tres niveles de dominio en grado y máster. En estas rúbricas se establece el/los resultado/s de aprendizaje en función de cada nivel de dominio y para cada competencia transversal (Villa, 2007). En el caso de las asignaturas optativas de ámbito nuclear, nos interesan especialmente los siguientes resultados de aprendizaje por nivel de dominio:

1. Comprensión e integración:

- Dominio I: describir, relacionar e interpretar situaciones y planteamientos sencillos.

- Dominio II: proceder con lógica y relacionar e integrar conceptos en situaciones complejas.

- Dominio III: identificar las carencias de información y utilizar el conocimiento con un enfoque globalizador.

2. Aplicación y pensamiento práctico.

- Dominio I: aplicar a la práctica su capacidad y los recursos de los que dispone para alcanzar objetivos en situaciones habituales, siguiendo instrucciones.

- Dominio II: diseñar un plan coherente con acciones concretas para abordar situaciones nuevas con un enfoque propio

- Dominio III: diseñar un plan coherente en acciones concretas para abordar situaciones complejas de forma individual o en colaboración con otros.

3. Conocimiento de problemas contemporáneos:

- Dominio I: reconocer los problemas contemporáneos que afectan a su campo profesional.

- Dominio II: analizar los problemas contemporáneos que afectan a su campo profesional.

- Dominio III: valorar y tomar conciencia de los problemas contemporáneos que afectan a su campo profesional y campos

afines.

4. Instrumental específica.

- Dominio I: emplear correctamente las herramientas básicas del ámbito profesional de forma guiada.

- Dominio II: integrar correctamente las herramientas básicas del ámbito profesional de forma autónoma.

- Dominio III: integrar correctamente las herramientas avanzadas del ámbito profesional.

(c) EY-NC-ND 2017, Universitat Politècnica de València 
Puesto que todas las asignaturas de este estudio son de $4^{\circ}$ curso de Grado, se optado por trabajar los resultados de aprendizaje del nivel de dominio II.

\subsection{La coordinación en la optatividad nuclear}

La coordinación de la optatividad comienza por identificar los siguientes resultados de aprendizaje:

- Proceder con lógica y relacionar e integrar conceptos en situaciones complejas.

- Diseñar un plan coherente con acciones concretas para abordar situaciones nuevas con un enfoque propio

- Analizar los problemas contemporáneos que afectan a su campo profesional.

- Integrar correctamente las herramientas básicas del ámbito profesional de forma autónoma.

Es importante señalar que todas las acciones (relacionar, diseñar, analizar e integrar) se encuentran en un nivel similar de complejidad según la taxonomía de Bloom.

\subsection{Diseño, desarrollo y resultados de actividades}

El siguiente paso en la coordinación de la optatividad consiste en diseñar actividades adecuadas para trabajar y evaluar las competencias transversales objeto de este estudio. La idea es aprovechar y potenciar las sinergias entre las diferentes asignaturas para reforzar el trabajo, destacando la coherencia de planteamientos y estrategias de evaluación. Con este objetivo, es primordial programar reuniones entre los responsables antes y durante el periodo de impartición de estas asignaturas. En nuestro caso, puesto que todas las asignaturas se imparten en el cuatrimestre B, se propone una reunión de coordinación en el cuatrimestre A y dos reuniones en el B. Finalmente se aconseja realizar una reunión de coordinación al finalizar las asignaturas (en el mes de mayo). En la primera reunión de coordinación, los responsables de las asignaturas exponen qué actividades van a realizar para trabajar las diferentes competencias. Es en este momento cuando la coordinación cobra mayor importancia. El hecho de informar a los responsables de las asignaturas, de las actividades que vamos a realizar en las nuestras, permite diseñar estrategias comunes. En el presente curso, y en el ámbito que nos ocupa, se han diseñado tres actividades fundamentalmente, tal y como se expone a continuación.

En la asignatura de "Centrales Nucleares Avanzadas" se planifica mediante 4 sesiones de prácticas, la elaboración de un modelo del núcleo de un reactor ACR (Advance CANDU Reactor) con un código de Monte Carlo. Esta actividad es un claro ejemplo de trabajo en las competencias transversales de "Aplicación y Pensamiento Práctico" e "Instrumental específica". En este caso, los autores disponemos de cierta experiencia previa (Gallardo, 2015 y Villanueva, 2016) en el trabajo y evaluación de "Conocimiento de Problemas Contemporáneos". 
En la asignatura "Seguridad Nuclear" se simula mediante un código termohidráulico un accidente de pérdida de refrigerante en una central nuclear, trabajando con ello las competencias transversales de "Comprensión e Integración" e "Instrumental específica".

En la asignatura "Protección Radiológica" se propone la búsqueda, análisis y exposición de un accidente radiológico y sus consecuencias, trabajándose de esa manera la competencia transversal de "Comprensión e Integración".

Si analizamos con detenimiento las tres actividades por separado, podremos concluir que de manera natural, se está estudiando un mismo problema desde tres puntos de vista distintos. En el primer caso se estudia el diseño, en el segundo caso se estudia la seguridad y en el tercer caso, las consecuencias de un accidente. Con esta idea clara en mente, la coordinación se centra en aportar coherencia a los objetivos, metodología y resultados de las tres actividades para que el alumno perciba las componentes multidisciplinares de un mismo caso o de casos análogos, importando la propia filosofía del aprendizaje basado en problemas (Escribano, 2008).

Queda pues claro que los objetivos de la coordinación son los siguientes: presentar la actividad de una manera transversal relacionándola con las tres asignaturas, guiar al alumno de manera coherente y señalar los resultados relacionándolos con las competencias transversales de las tres asignaturas.

De esta manera, se llega a la conclusión de que se necesita que el diseño, desarrollo y resultados de las actividades debe ser compartida por los responsables de las tres asignaturas. En este contexto, se decide adaptar las rúbricas que originalmente se utilizan en las asignaturas para que incluyan algunos indicadores comunes a las tres.

\subsection{La evaluación de competencias transversales en la optatividad nuclear}

Una vez que la coordinación ha permitido identificar las relaciones entre las tres actividades y se ha establecido una estrategia común para guiar al alumno en la realización de la misma, es necesario realizar el mismo ejercicio de coherencia en la evaluación. Recordemos que el objetivo es realizar la evaluación teniendo en cuenta la capacidad para relacionar, integrar, diseñar y analizar contenidos en situaciones complejas utilizando herramientas adecuadas.

Este punto es especialmente delicado ya que implica realizar un ejercicio de abstracción que afecta a nuestra asignatura y a las otras asignaturas de la optatividad.

A modo de ejemplo se va a realizar la explicación de la metodología seguida en la evaluación sobre la competencia transversal de "Aplicación y pensamiento práctico" de la asignatura "Centrales Nucleares Avanzadas". En este caso concreto se parte de una rúbrica con la que se está trabajando en los último dos cursos. En dicha rúbrica se reflejan los siguientes indicadores:

1. Analiza lo que es y no es un problema y toma la decisión de abordarlo.

2. Lee $y / o$ escucha activamente. Hace preguntas para definir el problema planteado.

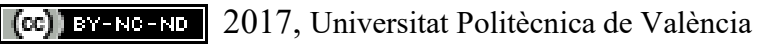


3. Recoge y analiza la información significativa que necesita para resolver los problemas en base a datos y no sólo a opiniones subjetivas y sigue un método lógico de análisis de la información.

4. Sigue un método lógico para identificar las causas de un problema y no quedarse en los síntomas.

5. Diseña un plan de acción para la aplicación de la solución escogida.

A partir de la coordinación entre las asignaturas de la optatividad ha quedado patente que queremos incluir en la evaluación referencias directas a las otras asignaturas. Con este objetivo, buscaremos mecanismos para incluir en alguno de los indicadores anteriores, la valoración a dicho indicador a partir de lo trabajado en las otras asignaturas.

En el caso del indicador 1, "Analiza lo que es y no es un problema y toma la decisión de abordarlo", para evaluarlo se ha pensado realizar un check-list que recoja explícitamente información de qué manera el problema que se está analizando, está relacionado con las asignaturas "Seguridad Nuclear" y "Protección Radiológica".

En el caso del indicador 3, "Recoge y analiza la información significativa...", se ha pensado incluir en el enunciado referencias bibliográficas e información general que el alumno puede (aunque no necesariamente) utilizar y que pertenece a las otras asignaturas. Por ejemplo, en el caso de la actividad del reactor ACR (en la asignatura de Centrales Nucleares Avanzadas), el objetivo es realizar el diseño, pero en el enunciado de la actividad se incluirá información de la seguridad y de los posibles accidentes y sus consecuencias. Recordemos que tanto la seguridad como el estudio de las consecuencias radiológicas son objeto de aprendizaje de las otras asignaturas optativas. De esta manera, el alumno debe manejar un mayor volumen de información y se ve obligado a cribarla discerniendo la que es relevante para la tarea, pero al mismo tiempo está realizando un ejercicio de integración de la información. El ejercicio de criba de información no es trivial, ya que en muchas ocasiones es muy complejo poder separar el diseño respecto de la seguridad y respecto de las implicaciones radiológicas.

En resumen, en el caso de "Centrales Nucleares Avanzadas", la evaluación de la competencia transversal de "Aplicación y pensamiento práctico" se realizará mediante una rúbrica de nivel de dominio II con 5 indicadores. Estos indicadores, a su vez, recogerán la información de diferentes preguntas planteadas en la actividad que no están directamente relacionadas con el objeto principal de la actividad, pero que es transversal con las otras asignaturas de la optatividad.

\section{Resultados}

Tal y como se ha explicado en el apartado 2.1, la optatividad de ámbito nuclear tiene actualmente un número reducido de alumnos matriculados (entre 3 y 7 dependiendo de la asignatura), por lo que la obtención de conclusiones desde el punto de vista de la significancia estadística es casi imposible. Conscientes de esta limitación, los autores de este trabajo consideramos que podemos aprovechar el hecho de tener baja matrícula para realizar entrevistas informales a los alumnos para poder obtener una retroalimentación de calidad. 
Con esta premisa, se elabora un pequeño cuestionario con el objetivo principal de saber si el alumno ha percibido o no que el trabajo realizado en el ámbito de las competencias transversales ha aportado un análisis "multi-dimensional" (es decir, de las diferentes asignaturas) y ha permitido ampliar el campo de visión del problema estudiado.

El cuestionario que se ha elaborado es el siguiente (a discutir informalmente en una entrevista):

- ¿Os hemos transmitido de forma adecuada que las tres asignaturas están íntimimamente relacionadas?

- ¿Crees que es bueno aislar un problema sin tener en cuenta las implicaciones y efectos no estudiados en la asignatura?

- ¿Crees que la actividad desarrollada es adecuada para la asignatura? ¿Ha sido buena idea incluir mucha más información que la que estrictamente necesitabas para llevarla a cabo?

- Con tus propias palabras, ¿qué crees que te ha aportado realizar esta actividad más allá de estudiar los contenidos de la asignatura?

- Desde el punto de toda la titución (GIE), ¿crees que esta actividad te ha servido para constatar la importancia de relacionar los conocimientos adquiridos a otros ámbitos de la profesión?

- ¿Has percibido que hemos trabajado de forma conjunta competencias mediante tres asignaturas?

- En general, ¿estás safisfecho con la actividad desarrollada?

En general, las entrevistas realizadas muestran que los alumnos perciben claramente que estamos trabajando un mismo contenido desde puntos de vista distintos y que esta diversidad de puntos de vista enriquece el mensaje final de la propia asignatura y refuerza la compactación y coherencia de contenidos, metodología y evaluación.

Pretendemos que esta experiencia no se quede en eso, una experiencia. Para ello, los trabajos futuros que vamos a realizar van a ir encaminados a ampliar la coordinación y el trabajo integrado de competencias al resto de asignaturas de ámbito nuclear, incluidas las de carácter obligatorio.

\section{Conclusiones}

En este trabajo se ha expuesto una primera experiencia realizada para mejorar la coordinación de las asignaturas de ámbito nuclear y carácter optativo en el Grado de Ingeniero de la Energía (GIE). La coordinación ha puesto de manifiesto que no siempre entendemos todos de igual manera el significado de una misma competencia tranversal y que es necesario unificar criterios para no trasladar la incertidumbre a los alumnos. Además de este primer beneficio, la coordinación en competencias transversales permite aprovechar sinergias entre diferentes asignaturas, evitando solapes y potenciando integración de conceptos que tradicionalmente transmitimos de manera atomizada a los alumnos. En general, la 
experiencia es muy positiva, recibiendo una buena acogida por parte de los alumnos y abriendo la puerta a ampliar esta coordinación al resto de asignaturas de contenido nuclear en GIE.

\section{Referencias}

UNIVERSITAT POLITÈCNICA DE VALÈNCIA, (2015a). Presentación de Competencias Transversales UPV CT-10. Conocimiento de problemas contemporáneos.

UNIVERSITAT POLITÈCNICA DE VALÈNCIA, (2015b). Rúbrica UPV CT-10. Conocimiento de problemas contemporáneos.

VILLA, A. y POBLETE, M. (2007), Aprendizaje basado en competencias. Una propuesta para la evaluación de las competencias genéricas, Universidad de Deusto, Bilbao

ESCRIBANO, A., DEL VALlE, A. (2008), El aprendizaje basado en problemas. Una propuesta metodológica en Educación Superior, Narcea, S.A. de Ediciones.

SANZ DE ACEO, M. L. (2010), Competencias cognitivas en Educación Superior, Narcea, Madrid.

GALLARDO, S., CARLOS, S. (2015), "Desarrollo de la competencia transversal de "Análisis y Resolución de Problemas" en la asignatura Centrales Nucleares Avanzadas", IN-RED, Valencia, Universitat Politècncia de València.

VILLANUEVA, J.F. et al. (2016), "Integración de la competencia transversal de "conocimiento de problemas contemporáneos" en Asignaturas de Grado", IN-RED, Valencia, Universitat Politècncia de València. 